\title{
Multiple coexistence equilibria in a two parasitoid-one host model
}

\author{
Ferdinand Pfab ${ }^{\mathrm{a}, *}$, Odo Diekmann ${ }^{\mathrm{b}}$, Souvik Bhattacharya ${ }^{\mathrm{a}}$, Andrea \\ Pugliese $^{\mathrm{a}}$ \\ ${ }^{a}$ Department of Mathematics, University of Trento, Italy \\ ${ }^{b}$ Department of Mathematics, Utrecht University, Netherlands
}

\begin{abstract}
Briggs et al. (1993) introduced a host-parasitoid model for the dynamics of a system with two parasitoids that attack different juvenile stages of a common host. Their main result was that coexistence of the parasitoids is only possible when there is sufficient variability in the maturation delays of the host juvenile stages. Here we analyse the phenomenon of coexistence in that model more deeply. We show that with some distribution families for the maturation delays, the coexistence equilibrium is unique, while with other distributions multiple coexistence equilibria can be found. In particular we find that stable coexistence does not necessarily require mutual invasibility.
\end{abstract}

Keywords: Population dynamics, Parasitoid-Host Interaction, Delay

Differential Equations, Multiplicity of coexistence equilibria 2000 MSC: 92-02

\section{Introduction}

2 It is known that parasitoid species of the same host can coexist (Force, 1970;

3 Price, 1970; Harvey et al., 2009). This observation seems to contradict a

4 principle in ecology which predicts that competing species cannot coexist on

5 the same limiting resource (Gause and Witt, 1935), though it has been shown

\footnotetext{
${ }^{*}$ Corresponding author

Email addresses: ferdinand.pfab@gmail.com (Ferdinand Pfab), o.diekmann@uu.nl (Odo Diekmann), souvik. bhattacharya@unitn.it (Souvik Bhattacharya), pugliese@science.unitn.it (Andrea Pugliese)
} 
that the principle holds under very stringent equilibrium conditions (Chesson and Case, 1986) and that competitors can coexist on the same biological resource along periodic solutions (Hsu et al., 1977; Armstrong and McGehee, 1980). Parasitoid species are a particularly interesting case, as various mechanisms that can promote parasitoid coexistence on the same host have been suggested (Price, 1970; Lane et al., 2006; Hackett-Jones et al., 2009). Briggs (1993) started to investigate under which conditions parasitoids can coexist when they attack different juvenile stages of a common host. This investigation was continued by Briggs et al. (1993), who found that in their model coexistence at equilibrium is possible only when there is sufficient variability in the maturation delays of the juvenile stages. They suggested that when the variability is large enough, different host individuals can be interpreted as different resources: individuals with a relatively long egg phase support the egg parasitoid, and individuals with a relatively long larva phase support the larva parasitoid. In the present paper we re-analyse the model by Briggs et al. (1993) and find more complex patterns than those already identified: there may be multiple coexistence equilibria, and, contrary to conventional wisdom, stable coexistence does not require mutual invasibility. The model is presented in Section 2. In Sections 3, 4 and 5 we formulate the original results in our somewhat different notation and in Section 6 show that coexistence equilibria are not unique for many distributions of the maturation delays. Finally, in Section 7 we set our results in the context of other works, discuss their relevance for biological pest control, and propose questions for further investigation. A general introduction to parasitoid-host systems can be found, for instance, in the text book by Godfray (1994).

\section{The model}

The model describes a host with two juvenile stages $E$ and $L$, and an adult stage $A$. We refer to the first juvenile stage as eggs and to the second juvenile stage as larvae but they can also represent other developmental stages as pupae or different instars. The egg stage is attacked by an egg parasitoid (whose density is denoted by $P$ ) while the larva stage is attacked by a larva parasitoid (density denoted by $Q$ ) with attack rates $a_{P}$ and $a_{Q}$ respectively. Non-infected host juveniles have random maturation delays which are distributed with probability density functions $w_{E}$ and $w_{L}$. Infected hosts do not progress to the next stage but give rise to new parasitoids a constant time $T_{J P}$ or $T_{J Q}$ after the infection. Unlike the original paper, we 
where with

$S_{E}\left(x_{E}, t\right)=\exp \left(-\int_{t-x_{E}}^{t}\left(a_{P} P(y)+d_{E}\right) d y\right)$

$$
\begin{aligned}
& R_{E}(t)=\rho d_{A} A(t) \\
& M_{E}(t)=\int_{0}^{\infty} R_{E}\left(t-x_{E}\right) S_{E}\left(x_{E}, t\right) w_{E}\left(x_{E}\right) d x_{E}
\end{aligned}
$$$$
M_{L}(t)=\int_{0}^{\infty} M_{E}\left(t-x_{L}\right) S_{L}\left(x_{L}, t\right) w_{L}\left(x_{L}\right) d x_{L}
$$

$$
S_{L}\left(x_{L}, t\right)=\exp \left(-\int_{t-x_{L}}^{t}\left(a_{Q} Q(y)+d_{L}\right) d y\right)
$$

host egg recruitment rate host egg maturation rate $=$ host larva recruitment rate host larva maturation rate $=$ host adult recruitment rate

probability for host eggs to survive from time $t-x_{E}$ to $t$

probability for host larvae to survive from time $t-x_{L}$ to $t$ 
60

\begin{tabular}{l|l}
$d_{Q}$ & background mortality rate of larva parasitoids \\
\hline$a_{P}$ & egg parasitoid attack rate \\
$a_{Q}$ & larva parasitoid attack rate \\
\hline$c_{P}$ & expected number of egg parasitoids emerging from infected egg \\
$c_{Q}$ & expected number of larva parasitoids emerging from infected larva \\
\hline$T_{J P}$ & duration of juvenile egg parasitoid stage \\
$T_{J Q}$ & duration of juvenile larva parasitoid stage
\end{tabular}

63 and

\begin{tabular}{l|l} 
function & description \\
\hline$w_{E}$ & probability density function for host egg maturation delay \\
$w_{L}$ & probability density function for host larva maturation delay
\end{tabular}

66

\section{Preliminaries}

In order to investigate equilibrium states, we introduce some quantities that 9 depend on constant parasitoid densities $P$ and $Q$. Note first that eggs and - larvae can have three different fates: they can die due to the background 71 death rates $d_{E}$ and $d_{L}$, they can be successfully attacked by parasitoids or 72 they can progress to the next stage. We first state the formulae for the ${ }_{3}$ transition probabilities between the host stages and the expected durations $7_{4}$ in the different stages (for the full computations see Appendix A).

75 The probability that a freshly emerged egg hatches into a larva is

$$
\Pi_{1}(P)=\int_{0}^{\infty} w_{E}(\tau) e^{-\left(a_{P} P+d_{E}\right) \tau} d \tau
$$

and the probability that a freshly hatched larva emerges as an adult is

$$
\Pi_{2}(Q)=\int_{0}^{\infty} w_{L}(\tau) e^{-\left(a_{Q} Q+d_{L}\right) \tau} d \tau .
$$


77 As shown in Appendix A.2, the expected duration of the egg stage is

$$
\Gamma_{1}(P)=\frac{1-\Pi_{1}(P)}{a_{P} P+d_{E}}
$$

the expected duration of the larva stage (given that this stage is reached) is

$$
\Gamma_{2}(Q)=\frac{1-\Pi_{2}(Q)}{a_{Q} Q+d_{L}}
$$

79 80 is is

nd the expected duration of the adult stage (given that this stage is reached)

$$
\Gamma_{3}=\frac{1}{d_{A}}
$$
$\left.{ }_{89} \quad \frac{d L}{d t}=0\right)$,

We now can state the following relations, valid when the related population densities are constant:

The rate of eggs emerging, given constant adult density $A$, is by definition

$$
R_{E}=\rho d_{A} A
$$

The constant egg density $E$ is the product of the rate of eggs emerging and the expected duration of the egg stage (to verify set $\frac{d E}{d t}=0$ ),

$$
E=R_{E} \Gamma_{1}(P) .
$$

The constant larva density $L$ is the product of three factors, viz., the rate of eggs emerging, the probability for an egg to mature to a larva and the expected duration of the larva stage, given that it is reached (to verify set

$$
L=R_{E} \Pi_{1}(P) \Gamma_{2}(Q) .
$$

The constant adult density $A$ is the product of four factors, viz., the rate of eggs emerging, the probability for an egg to mature to a larva, the probability for a larva to mature to an adult and the expected life length of an adult (to verify set $\left.\frac{d A}{d t}=0\right)$,

$$
A=R_{E} \Pi_{1}(P) \Pi_{2}(Q) \Gamma_{3} .
$$

94 The average number of offspring from a freshly laid egg (the basic reproduction number of the host) is the product of the average output of an adult $\rho$ and the probability for an egg to mature to an adult,

$$
R_{0}=\rho \Pi_{1}(P) \Pi_{2}(Q) .
$$


At a nontrivial equilibrium the basic reproduction number $R_{0}$ equals one, as can be seen by plugging the definition of $R_{E}$ into equation (10). The zero growth condition for host eggs (8) and larvae (9) can be combined by eliminating $R_{E}$. This yields

$$
\frac{\Pi_{1}(P) \Gamma_{2}(Q)}{\Gamma_{1}(P)}=\frac{L}{E} .
$$

\section{Equilibrium states}

\subsection{When only the egg parasitoid is present}

For the case that only the egg parasitoid is present, its equilibrium density $P^{*}$ can be determined by plugging $Q=0$ into the basic reproduction number $R_{0}$, which is equal to 1 at equilibrium, i.e. by requiring

$$
\rho \Pi_{1}\left(P^{*}\right) \Pi_{2}(0)=1 .
$$

Assuming that $R_{0}>1$ for $P=0$ and $Q=0$, this equation has a unique root for $P^{*}$ since $R_{0}$ approaches 0 strictly monotonically with increasing $P$.

The equilibrium state for the egg density is determined by the requirement of zero growth rate for (non-trivial) $P$. This, by setting $d P(t) / d t=0$ and assuming constant population densities, leads to

$$
E_{P}^{*}=\frac{d_{P}}{a_{P} c_{P}} .
$$

The equilibrium larva density $L_{P}^{*}$ in presence of only the egg parasitoid can be calculated from the relation (12),

$$
L_{P}^{*}=E_{P}^{*} \frac{\Pi_{1}\left(P^{*}\right) \Gamma_{2}(0)}{\Gamma_{1}\left(P^{*}\right)} .
$$

The host adult density can be obtained for all equilibrium systems by combining (7) and (8).

\subsection{When only the larva parasitoid is present}

In the same way as for the egg parasitoid, we can derive the equilibrium densities for the case that only the larva parasitoid is present. The equilibrium larva parasitoid density $Q^{*}$ is determined through the equation

$$
\rho \Pi_{1}(0) \Pi_{2}\left(Q^{*}\right)=1
$$


and again this equilibrium density is unique. The equilibrium larva density is

$$
L_{Q}^{*}=\frac{d_{Q}}{a_{Q} c_{Q}},
$$

and the equilibrium egg density is

$$
E_{Q}^{*}=L_{Q}^{*} \frac{\Gamma_{1}(0)}{\Pi_{1}(0) \Gamma_{2}\left(Q^{*}\right)} .
$$

\subsection{When both parasitoids are present}

According to equation (11) the host adult density is in equilibrium when the parasitoid densities satisfy

$$
Q=\Pi_{2}^{-1}\left(\frac{1}{\rho \Pi_{1}(P)}\right)
$$

126 where $\Pi_{2}^{-1}$ is the inverse function of $\Pi_{2}$. Plugging (19) into (12) yields a ${ }_{127}$ condition for all host stages to be in equilibrium

$$
f(P)=\frac{L}{E}
$$

where $f:\left[0, P^{*}\right] \rightarrow \mathbb{R}^{+}$is defined by

$$
f(P)=\frac{\Pi_{1}(P)}{\Gamma_{1}(P)} \Gamma_{2}\left(\Pi_{2}^{-1}\left(\frac{1}{\rho \Pi_{1}(P)}\right)\right) .
$$

When both parasitoids coexist, the equilibrium egg and larva densities are determined by the requirement of zero growth rate for the egg and larva parasitoid respectively. Hence they are given by $E_{P}^{*}$ and $L_{Q}^{*}$, and thus the egg parasitoid coexistence equilibrium $P^{* *}$ is determined by the condition

$$
f\left(P^{* *}\right)=\frac{L_{Q}^{*}}{E_{P}^{*}}
$$

3. The corresponding larva parasitoid density $Q^{* *}$ can be obtained by equation (19).

Note that in the same way one can derive an equivalent function $g(Q)=L / E$ 
which determines coexistence equilibria by $g\left(Q^{* *}\right)=\frac{L_{Q}^{*}}{E_{P}^{*}}$, where

$$
\begin{aligned}
g(Q) & =\frac{\Pi_{1}\left(\Pi_{1}^{-1}\left(\frac{1}{\rho \Pi_{2}(Q)}\right)\right)}{\Gamma_{1}\left(\Pi_{1}^{-1}\left(\frac{1}{\rho \Pi_{2}(Q)}\right)\right)} \Gamma_{2}(Q) \\
& =\frac{\Gamma_{2}(Q)}{\Pi_{2}(Q)} \frac{1}{\rho \Gamma_{1}\left(\Pi_{1}^{-1}\left(\frac{1}{\rho \Pi_{2}(Q)}\right)\right)}
\end{aligned}
$$

with $\Pi_{1}^{-1}$ being the inverse function of $\Pi_{1}$. All further analysis could be carried out with either $f$ or $g$ but for simplicity we stick with the function $f$. Turning back to the function $f$, we see that the shape of the function contains information on the multiplicity of coexistence equilibria. According to equation (22), multiple coexistence equilibria cannot arise if $f$ is strictly monotonic. If on the other hand for some parameters $f$ is not monotonic, we can always find values of the parameters $c_{P}, c_{Q}, d_{P}$ or $d_{Q}$ that give rise to multiple coexistence equilibria by shifting the critical horizontal $L_{Q}^{*} / E_{P}^{*}=d_{Q} a_{P} c_{P} / d_{P} a_{Q} c_{Q}$ until the graph of the function $f$ (which does not depend on those parameters) is intersected multiple times. Each intersection yields a coexistence equilibrium. Similarly, the critical horizontal can be shifted using those parameters until there are no coexistence equilibria.

\section{Invasibility of stable equilibria}

When in the absence of parasitoids $R_{0}>1$, either parasitoid can establish a population. Often, a stable host-parasitoid equilibrium will be reached with $R_{0}$ set at 1 (Murdoch et al., 1987) and we follow Briggs et al. (1993) in examining when this equilibrium can be invaded by the other parasitoid. A case where the host and parasitoid populations settle into a periodic solution is examined numerically in the next Section.

It is not difficult to show that a stable equilibrium population with only the larva parasitoid can be invaded by the egg parasitoid when the egg parasitoid alone reduces the egg density more than the larva parasitoid alone, that is when

$$
E_{P}^{*}<E_{Q}^{*}
$$

To demonstrate this, we compute the Malthusian parameter $\lambda=\lambda_{P}(E)$ for the egg parasitoid at constant egg density $E$. Namely, we linearise system 
162

and obtain

$$
\frac{d P(t)}{d t}=a_{P} c_{P} E P\left(t-T_{J P}\right)-d_{P} P(t)
$$

where $E=E_{Q}^{*}$. We then assume

$$
P(t)=e^{\lambda t} P(0)
$$

$$
\begin{aligned}
\lambda P(t) & =a_{P} c_{P} E P(t) e^{-\lambda T_{J P}}-d_{P} P(t) \\
\lambda & =E a_{P} c_{P} e^{-\lambda T_{J P}}-d_{P} .
\end{aligned}
$$

The egg parasitoid can invade a stable equilibrium community of the larva parasitoid and the host when this equation has a positive real root for $E=E_{Q}^{*}$, that is $\lambda_{P}\left(E_{Q}^{*}\right)>0$. The claim that this requires $E_{P}^{*}<E_{Q}^{*}$ follows because the unique real root $\lambda_{P}(E)$ increases strictly monotonically with $E$ and $\lambda_{P}\left(E_{P}^{*}\right)=0$. (Note that we do not have to consider complex roots for $\lambda$ since their real parts cannot exceed the real root.)

In the same way it can be seen that the larva parasitoid can invade a stable equilibrium population with only the egg parasitoid when

$$
L_{Q}^{*}<L_{P}^{*}
$$

173 We speak of mutual invasibility of stable equilibria when

$$
E_{P}^{*}<E_{Q}^{*} \text { and } L_{Q}^{*}<L_{P}^{*}
$$

The value of the function $f$ defined in (21) at the boundary of its domain, relative to the right hand side of (22), turns out to be related to the invasibility conditions. Indeed,

$$
\begin{aligned}
f(0) & =\frac{\Pi_{1}(0)}{\Gamma_{1}(0)} \Gamma_{2}\left(\Pi_{2}^{-1}\left(\frac{1}{\rho \Pi_{1}(0)}\right)\right) \\
& =\frac{\Pi_{1}(0)}{\Gamma_{1}(0)} \Gamma_{2}\left(\Pi_{2}^{-1}\left(\Pi_{2}\left(Q^{*}\right)\right)\right) \\
& =\frac{\Pi_{1}(0)}{\Gamma_{1}(0)} \Gamma_{2}\left(Q^{*}\right) \\
& =\frac{L_{Q}^{*}}{E_{Q}^{*}}
\end{aligned}
$$




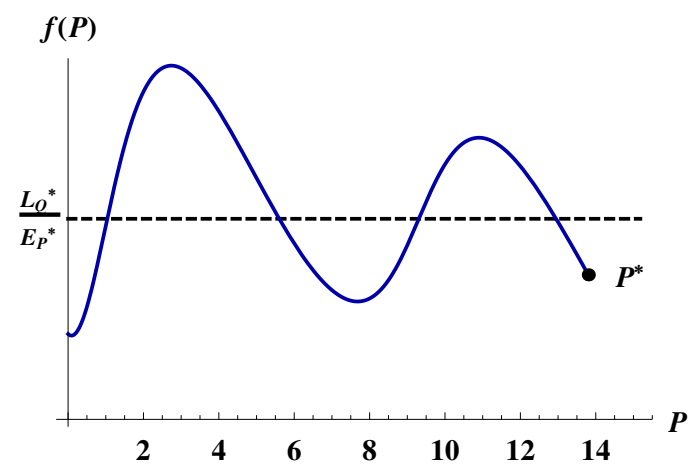

Figure 1: The graph of the function $f$ which intersects the level $L_{Q}^{*} / E_{P}^{*}$ when the egg parasitoid density corresponds to a coexistence equilibrium. The maturation delays are distributed with two discrete values each (see Appendix B.2). Parameter values are $T_{E_{1}}=0.2, T_{E_{2}}=1.35, T_{L_{1}}=0.75, T_{L_{2}}=5, r_{E}=0.5, r_{L}=0.3, a_{P}=2, a_{Q}=0.2$, $d_{E}=0, d_{L}=0, \rho=500, d_{P}=8, d_{Q}=0.175, c_{P}=0.5$ and $c_{Q}=0.5$

and

$$
\begin{aligned}
f\left(P^{*}\right) & =\frac{\Pi_{1}\left(P^{*}\right)}{\Gamma_{1}\left(P^{*}\right)} \Gamma_{2}\left(\Pi_{2}^{-1}\left(\frac{1}{\rho \Pi_{1}\left(P^{*}\right)}\right)\right) \\
& =\frac{\Pi_{1}\left(P^{*}\right)}{\Gamma_{1}\left(P^{*}\right)} \Gamma_{2}\left(\Pi_{2}^{-1}\left(\Pi_{2}(0)\right)\right) \\
& =\frac{\Pi_{1}\left(P^{*}\right)}{\Gamma_{1}\left(P^{*}\right)} \Gamma_{2}(0) \\
& =\frac{L_{P}^{*}}{E_{P}^{*}}
\end{aligned}
$$

which implies that the egg parasitoid can invade a stable equilibrium with the larva parasitoid alone when $f(0)<L_{Q}^{*} / E_{P}^{*}$ and the larva parasitoid can invade a stable equilibrium with the egg parasitoid alone when $f\left(P^{*}\right)>L_{Q}^{*} / E_{P}^{*}$.

\section{Applying distributions for the maturation delays}

We apply several distributions for the maturation delays in order to analyze their influence on the multiplicity of coexistence equilibria. Among those are the constant-duration distribution, (shifted) exponential distribution and (shifted) gamma distribution, which have been introduced in the original paper of Briggs et al. (1993). Here the term 'shifted' refers to including minimal 
values for the maturation delays. Additionally we introduce a two-value distribution where the maturation delays assume one of two discrete values with certain probabilities.

It turns out that, among these distributions, only the constant-duration and the (non-shifted) exponential distribution yield at most one coexistence equilibrium. For those two distributions the function $f$ is monotonic and therefore the critical horizontal $L_{Q}^{*} / E_{P}^{*}$ can be crossed at most once. Elementary representations for $f$ in those cases are shown in Appendix B. For the case of constant maturation delays, $f$ is decreasing and hence there is a coexistence equilibrium only if $f(0)>L_{Q}^{*} / E_{P}^{*}>f\left(P^{*}\right)$, implying that neither parasitoid can invade a stable equilibrium of the other parasitoid and the host. For the case of exponentially distributed maturation delays, $f$ is increasing and hence, in the other way around, there must be mutual invasibility of stable equilibria for a coexistence equilibrium to exist.

For all the other distributions (two-value distribution, shifted exponential distribution and (normal or shifted) gamma distribution), we could numerically find parameters so that the graph of $f$ crosses the critical horizontal line multiple times, giving rise to multiple equilibria. Fig. 1 shows an example where the graph of $f$ crosses the critical horizontal line four times with two-value distributions for the maturation delays (see caption).

\subsection{Simulations and stability}

To see how the system behaves after a small perturbation from an equilibrium, we computed time plots with the software Mathematica shown in Fig. 2. The plots reveal that coexistence equilibria can be stable or unstable, possibly giving rise to oscillations around the equilibrium after perturbation. Bifurcation diagrams are shown in Fig. 3. The left panel shows how the parameter $a_{P}$ shifts the horizontal in Fig. 1 without changing the function $f$, and thus we can observe how coexistence equilibria appear and disappear in pairs when changing the parameter. The right panel shows how the adult mortality $d_{A}$ affects stability without changing the equilibrium values (since this parameter does not occur in the function $f$ or in the level of the critical horizontal line). Low values for $d_{A}$ seem to stabilize some equilibria while high values for $d_{A}$ appear to destabilize all equilibria.

We further analyzed the dynamics for low values of the host adult death rate $d_{A}$. We show some simulations for that case in the $(P, Q)$-plane in Fig. 4. There we see that the population densities lie on the curve of equation 
I

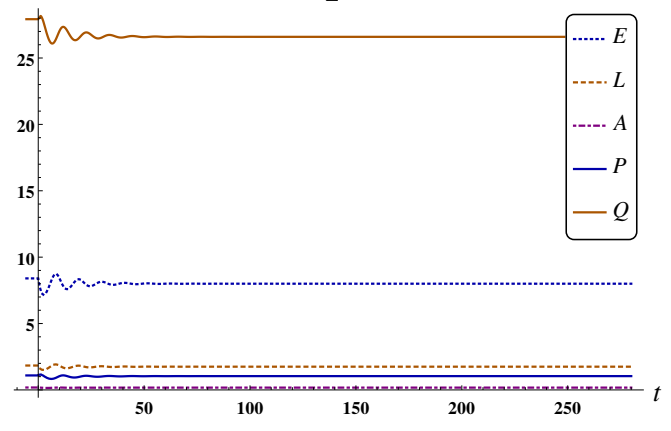

III

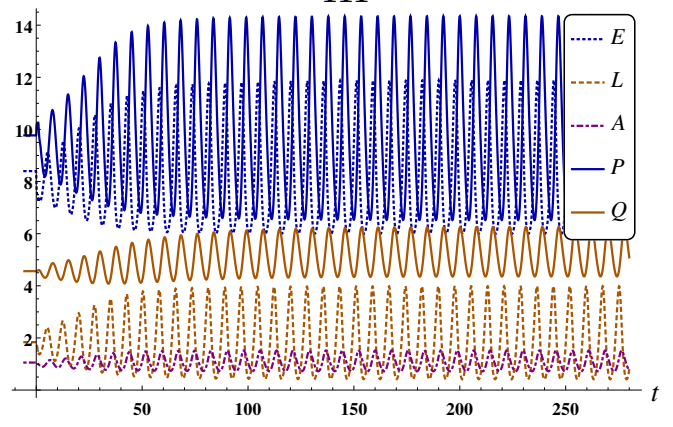

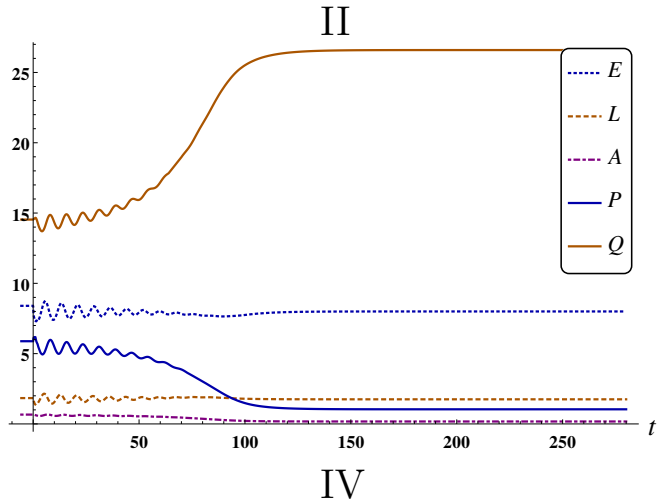

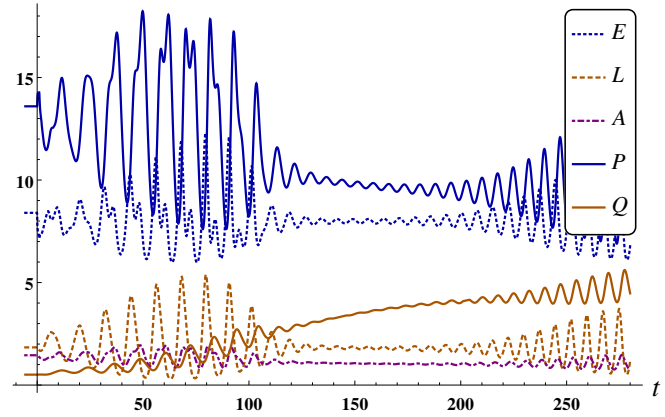

Figure 2: Time plots of population dynamics after small perturbations from equilibrium densities. The initial densities for $t \leq 0$ are constant and correspond to perturbations from the equilibrium densities indicated by the first (I), second (II), third (III) and forth (IV) intersection of the graph of $f$ with the critical horizontal in Fig. 1. The perturbations consist of increasing all equilibrium densities by $5 \%$. Note that in plot (IV) the same attractor as in plot (III) seems to be approached. Distributions and parameter values are the same as in Fig. 1 . Additionally $T_{J P}=1, T_{J Q}=1$ and $d_{A}=0.3$ $\Pi_{1}(P) \frac{\Gamma_{2}(Q)}{\Gamma_{1}(P)}=\frac{L_{Q}^{*}}{E_{P}^{*}}$ and move in a direction depending on the relative position of this curve and the curve $\rho \Pi_{1}(P) \Pi_{2}(Q)=1$. This can be justified through a time-scale argument that we just sketch here, leaving details to future work. For the argument note that $A(t)$ is a slow variable when $d_{A}$ is low, what can be seen from the models definition (1); thus in the fast time-scale $E(t), L(t), P(t)$ and $Q(t)$ will evolve under a constant value for the rate of eggs emerging, see equation (7). Numerical evidence suggests that this reduced system always quickly converges to its (quasi)-equilibrium, where $E=E_{P}^{*}, L=L_{Q}^{*}$ and equations (8) and (9) hold, corresponding to the solid curve in the $(P, Q)$-plane in Fig. 4 . Thus, on the slow time-scale, 

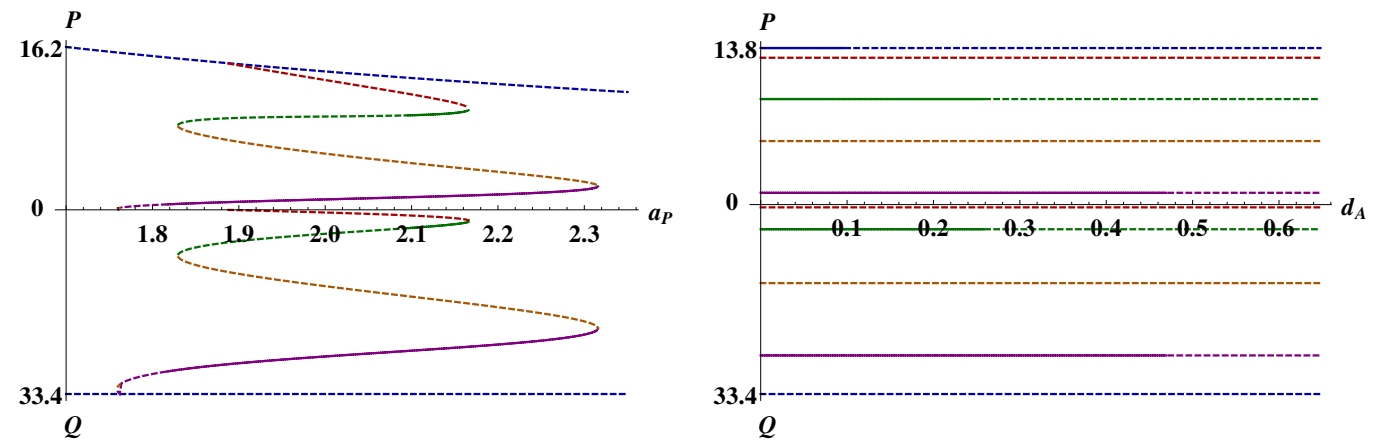

Figure 3: Bifurcation diagrams showing the equilibrium values for both parasitoid species. The upper vertical axis represents values for $P$ and the lower vertical axis represents values for $Q$. The outermost lines represent equilibria with only one parasitoid species while the inner equilibria are true coexistence equilibria. Values for $P$ and $Q$ corresponding to the same coexistence equilibrium are drawn with the same color in the online version of the article. Stability is indicated by solid (stable) and dashed (unstable) lines. For the stability analysis the eigenvalues of the characteristic equation were calculated with the MATLAB package eigAM/eigTMN by Breda et al. (2014). Parameter values are the same as in Fig. 1 and Fig. 2 (except axis parameters)

$A(t)$ changes according to the third equation of the system (1) with all other state variables at the quasi-equilibrium. It can be easily verified that $A(t)$ will increase or decrease according to whether the basic reproduction number $R_{0}$ from equation (11) is greater or smaller than 1 , thus according to whether $(P, Q)$ is above or below the dashed curve in Fig. 4 . As at the quasiequilibrium $A$ and $P$ are related by relation (8) with $E=E_{P}^{*}$, an increase [decrease] of $A(t)$ corresponds to an increase [decrease] of $P(t)$. This explains why the dynamics in the $(P, Q)$-plane is towards the right when the dashed curve is above the solid curve $\left(R_{0}>1\right)$ and towards the left when the dashed curve is below. Since the intersections between the two curves correspond to values of $(P, Q)$ where all state variables are at equilibrium, the previous graphical argument shows that, in the limit of $d_{A} \rightarrow 0$, an equilibrium is stable when the dashed curve crosses the solid curve from above, while it is unstable when the curves cross in the opposite way.

These findings can be transfered to the shape of the function $f(P)$. Indeed, it can be easily verified that the solid curve is below the dashed curve if and only if $f(P)$ is below $L_{Q}^{*} / E_{P}^{*}$. Therefore the findings above imply that coexistence equilibria are stable, for $d_{A}$ sufficiently small, when $f^{\prime}\left(P^{* *}\right)>0$, 

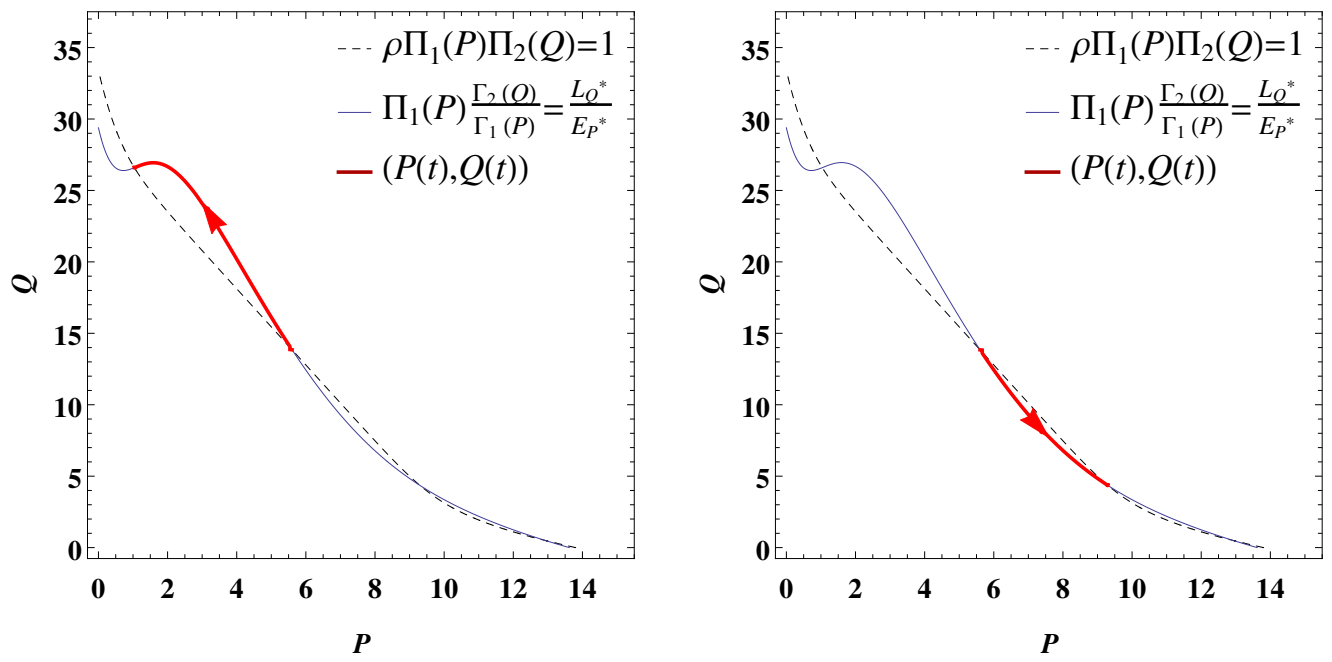

Figure 4: Parasitoid phase plane with time dynamics. Initial population densities (for $t \leq 0)$ correspond to the second coexistence equilibrium from left in Fig. 1. Perturbation is introduced via the host adult densities, which are respectively to its equilibrium value decreased by $1 \%$ in the left panel and increased by $1 \%$ in the right panel. Host adult mortality rate is very low, $d_{A}=0.001$. All other parameter values are as in Fig. 1 and 2

while they are unstable when $f^{\prime}\left(P^{* *}\right)<0$. The second statement appears to be true for all $d_{A}>0$ but we give a formal proof only for the scenario with constant maturation delays in Appendix C.2.

Further investigations of invasibility are illustrated with time plots in Fig. 5, where the larva parasitoid is introduced at very low density into an equilibrium system of egg parasitoid and host. This numerical example has important implications concerning invasion and coexistence which go beyond what was found by Briggs et al. (1993). One point is that the invasibility criteria stated in Section 5 do hold only for constant equilibria. If a single-parasitoid equilibrium is unstable with respect to the interaction of this parasitoid and the host, it has no sense to investigate its invasibility by the other parasitoid. Instead one should (numerically) find the single parasitoid-host attractor and investigate its invasibility (Metz et al., 1992). One may actually do this in one go by using the introduction of the second parasitoid as a way to perturb the unstable equilibrium as done in Fig. 5. As this figure reveals, oscillations may facilitate successful invasion in the sense that the second parasitoid is successful when the single parasitoid equilibrium is unstable $\left(d_{A}=0.3\right)$, while being unsuccessful in case it is stable $\left(d_{A}=0.05\right)$ since $L_{P}^{*}<L_{Q}^{*}$. By 


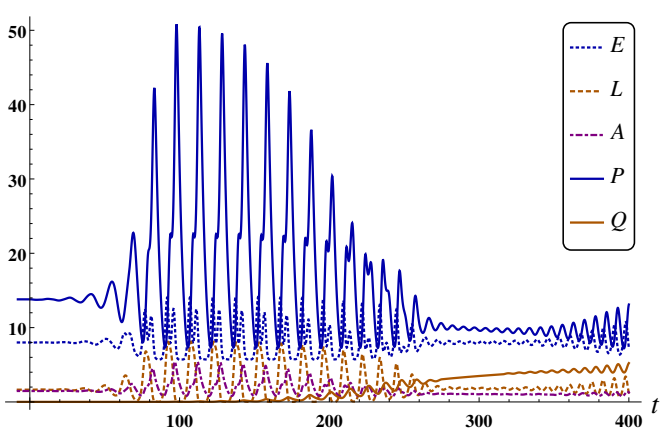

(a) For $d_{A}=0.3$ the equilibrium of host and egg parasitoid is unstable and the larva parasitoid can invade through oscillations

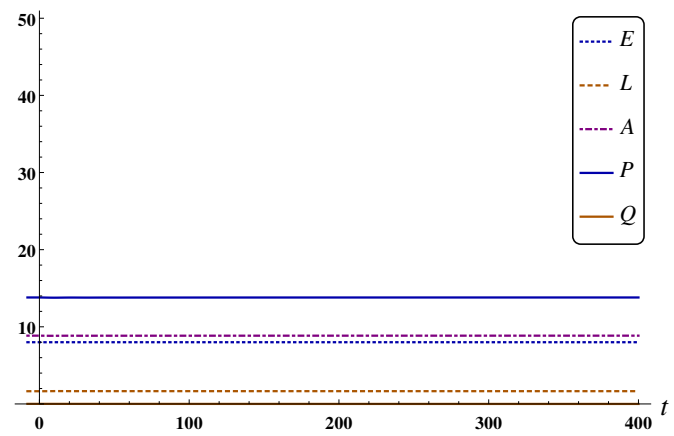

(b) For $d_{A}=0.05$ the equilibrium of host and egg parasitoid is stable and the larva parasitoid cannot invade

Figure 5: Time plots of population dynamics after introducing the larva parasitoid into an equilibrium system of egg parasitoid and host. The system is started with constant population densities for $t \leq 0$ corresponding to the equilibrium densities of egg parasitoid and host with additional a low density $Q=0.01$ of the larva parasitoid. Distributions and parameter values are the same as in Fig. 1 and 2 with exception of a lower value for $d_{A}$ in the right panel

combining Fig. 5b with the right panel of Fig. 3 another conclusion emerges: non-invasibility of a stable single-parasitoid equilibrium does not exclude the possibility of stable equilibrium coexistence of the two parasitoids (indeed, for $d_{A}=0.05$ we observe in Fig. 3 that simultaneously the equilibrium with only the egg parasitoid, and two coexistence equilibria are stable).

\section{Discussion}

We found multiple (non-trivial) coexistence equilibria in a model for the population dynamics of two parasitoids attacking different juvenile stages of a common host. The model was introduced by Briggs et al. (1993) and it involves distributed maturation delays for the host juvenile stages. We have shown that, depending on the distributions of the maturation delays, multiple coexistence equilibria can arise. To our knowledge, this is the first documented example of multiple coexistence equilibria in a parasitoid-host model, as well as the first example for the multiplicity of coexistence equilibria to depend on the distribution of maturation delays. 
Non steady-state attractors in parasitoid-host systems, in contrast, have received considerable attention before. Already the dynamics of the classical discrete-time model by Nicholson and Bailey (1935) are known to be oscillatory: one or both species go extinct after diverging oscillations around the unstable coexistence equilibrium. In a continuous-time parasitoid-host model by Murdoch et al. (1987), stability of a steady-state coexistence attractor can be facilitated by an invulnerable host stage. For modifications of this model, multiple non steady-state attractors have been found by Murdoch et al. (1992, 1997), Briggs (1993) and Briggs et al. (1999). Particularly Briggs (1993) shows that such non steady-state attractors can lead to parasitoid coexistence in situations where no stable coexistence equilibrium is predicted. Further Sieber and Hilker (2011) report multiple (non-)equilibrium attractors in a single host population that is exploited by microparasites and predators. Beyond that, there is a well-developed body of theory on coexistence in variable environments (deterministic and stochastic), see for example the works by Abrams (1984), Chesson (1994) and Li et al. (2016). Occurrence of oscillations in real parasitoid populations is documented by Godfray and Hassell (1989), who offer a review on oscillations of host parasitoid systems in the tropics and corresponding discrete and continuous models.

In our model we found that equilibria can have different properties. Single parasitoid equilibria are potentially stable and non-invadable only when the host stage of the other parasitoid is reduced more strongly than what would be needed by the competitor to sustain. Similarly we found that twoparasitoid coexistence equilibria are "potentially stable" only when increasing a parasitoid species reduces its own host stage relatively to its competitors host stage when the competing parasitoid species is chosen accordingly so that the host stays at equilibrium. Coexistence equilibria for which this is not the case turned out to be always unstable. This can be interpreted as a manifestation of the principle that coexistence of competitors can be possible only when intraspecific competition is stronger than interspecific competition, see for example the review by Chesson (2000). For the potentially stable equilibria we found that stability can be always altered with the parameter $d_{A}$ of host adult mortality (which does not change the equilibrium values due to the way the model is parameterized). Especially, we found that low values for $d_{A}$ generally stabilize potentially stable equilibria. In the other way around we found that high values for $d_{A}$ are always destabilizing. This is similar to the observations of Murdoch et al. (1987), who found for a similar single-parasitoid model that stable equilibria can exist only when there is a 
sufficiently long invulnerable adult stage of the host.

We made several observations concerning invasibility and single-parasitoid equilibria in the model. One point is that in the presence of multiple coexistence equilibria, stable coexistence can occur without mutual invasibility. We described a situation where the parasitoids can coexist although the larva parasitoid cannot invade a stable equilibrium of egg parasitoid and host $\left(L_{P}^{*}<L_{Q}^{*}\right)$. This is similar to the findings of Buonomo and Cerasuolo (2014) in a model for plants and parasites. Our example also shows that host juvenile densities can increase when an additional parasitoid is introduced since the equilibrium larva density with the egg parasitoid alone $L_{P}^{*}$ is lower than the equilibrium larva density $L_{Q}^{*}$ when both parasitoids coexist. Analogous examples can be found for situations where introducing the egg parasitoid increases the equilibrium egg density. These findings differ from those of other authors including Briggs (1993) and Briggs et al. (1993), who assume that stable coexistence requires mutual invasibility, and conclude that (in the absence of other mechanisms such as hyperparasitism) introducing a second parasitoid cannot lead to higher equilibrium densities of host juveniles. This is interesting in the light of the discussion whether single or multiple parasitoids should be introduced for optimal biological pest control, see for example the contributions by Ehler (1990) and Pedersen and Mills (2004). Furthermore we found that the invasibility criterion suggested by Briggs et al. (1993) is not generally valid when there are multiple coexistence equilibria. The original criterion states that a parasitoid species can invade only if its growth rate is positive at the equilibrium host density set by the resident parasitoid. We found however that if there are multiple coexistence equilibria, and the residents single-parasitoid equilibrium is not stable, invasion of the other parasitoid can take place through oscillations eventually leading to coexistence of both parasitoids. This is related to the findings on invasion in oscillating conditions by Armstrong and McGehee (1980), Bacaër and Guernaoui (2006), Greenman and Norman (2007) and Bate and Hilker (2013). Since in our model such situations occurred only when there are multiple coexistence equilibria we conjecture that this is indeed a necessary condition. The question remains of when coexistence equilibria can arise generally and what is the connection to the maturation delays of the hosts. A literature search reveals that the occurrence of multiple equilibria in population models is generally connected to some non-linearity or non-monotonicity in the interaction of different species. Evidence for that can be found in several models based on ordinary differential equations. Pimenov et al. (2015) find that in a 

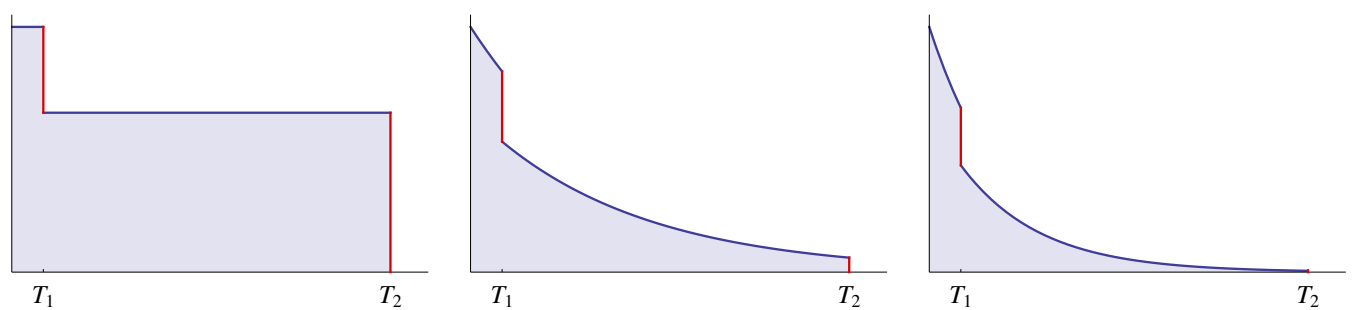

Figure 6: Age distribution of a host juvenile stage subjected to constant parasitism pressure $a_{P} P$ or $a_{Q} Q$ equal to $0,0.2$ or 0.4 (from left to right; note that there is no background death rate). The maturation delay for the stage is distributed by two discrete values $T_{1}$ and $T_{2}$ which occur with probabilities $r$ and $1-r$. The area under the curve represents the expectation value $\Gamma_{i}$ for the time in this stage, while the sum of the lengths of the vertical bars at the times $T_{1}$ and $T_{2}$ represents the probability $\Pi_{i}$ to reach the next stage. The ratio $\Gamma_{i} / \Pi_{i}$ equals approximately 8.8, 9.5 and 7.9 from left to right, and thus first increases and then decreases with increasing parasitism. Parameter values are: $r=0.35$, $T_{1}=1, T_{2}=12$

predator-prey model, multiple coexistence equilibria can arise when the prey changes its behavior in dependence of the predator density. Similarly Freeze et al. (2014) find multiple coexistence equilibria in a three species model where a super predator changes feeding behavior in dependence of its prey species densities. Buonomo and Cerasuolo (2014) find multiple coexistence equilibria in a model with host plants that react to parasitism in a non-linear way.

We found in our model too that multiple coexistence equilibria can occur only when the host larva-egg proportion depends in a non-monotonic way on the density of one parasitoid while the other parasitoid density is kept so that the host stays at equilibrium. We have seen that this can never happen for two important special cases: constant and exponentially distributed maturation delays. For constant maturation delays, increasing one parasitoid (and decreasing the other parasitoid accordingly) increases its own host stage relatively to the host stage of the competitor, which additionally implies that if there is a coexistence equilibrium, it is unstable and neither parasitoid can invade a stable population with the other parasitoid. Conversely for exponentially distributed maturation delays, increasing a parasitoid (and again decreasing the other parasitoid accordingly) reduces its host stage relatively to the host stage of the competitor, which additionally implies pairwise invasibility when there is a coexistence equilibrium. For all other distributions we investigated, the parasitoid densities can affect the hosts larva-egg proportion 
in a non-monotonic fashion giving rise to multiple coexistence equilibria. An illustration of how this can happen with the two-value distributions we used in our numerical examples is shown in Fig. 6. There we show the expected duration $\Gamma_{i}$ of a juvenile stage and the probability $\Pi_{i}$ to reach the next stage, both for different densities of the corresponding parasitoid. We see that increasing the parasitoid density first decreases $\Pi_{i}$ heavily because only a small part of the hosts with long maturation delay reaches maturation, while further increasing the parasitoid density decreases $\Gamma_{i}$ more strongly because parasitism still mainly affects hosts with a long maturation delay whose contribution to $\Pi_{i}$ was already low. Such mechanisms can lead to a non-monotonic relation between the parasitoid densities and the hosts larvaegg proportion, what potentially gives rise to multiple coexistence equilibria. Note however that the ratio of a parasitoids host stage and the other parasitoids host stage is according to (21) and (23) not only proportional to $\Gamma_{i} / \Pi_{i}$ but depends also on $\Gamma_{j}\left(\Pi_{j}^{-1}\left(\frac{1}{\rho \Pi_{i}}\right)\right.$ ) (where $j$ refers to the other parasitoids host stage); thus this graphical illustration is incomplete, but still, in our view, sheds some light on the mechanisms through which the distribution of maturation delays affects coexistence equilibria.

\section{Appendix A. Transition probabilities and expected duration of the stages}

Here we derive formulas for the transition probabilities from egg to larva $\Pi_{1}(P)$ and from larva to adult $\Pi_{2}(Q)$, and for the expected duration of the egg, larva and adult stage, $\Gamma_{1}(P), \Gamma_{2}(Q)$ and $\Gamma_{3}$ respectively. The calculations are valid for constant parasitoid densities $P$ and $Q$. We use the following notations for the various random variables 


\begin{tabular}{|c|c|c|}
\hline random variable & density & description \\
\hline$X_{E}$ & $w_{E}(\tau)$ & $\begin{array}{l}\text { time needed for egg matura- } \\
\text { tion }\end{array}$ \\
\hline$X_{L}$ & $w_{L}(\tau)$ & $\begin{array}{l}\text { time needed for larva matura- } \\
\text { tion }\end{array}$ \\
\hline$K_{E}$ & $\left(a_{P} P+d_{E}\right) e^{-\tau\left(a_{P} P+d_{E}\right)}$ & $\begin{array}{l}\text { time until an egg dies or is in- } \\
\text { fected (when it does not ma- } \\
\text { ture before), distributed ex- } \\
\text { ponentially }\end{array}$ \\
\hline$K_{L}$ & $\left(a_{Q} Q+d_{L}\right) e^{-\tau\left(a_{Q} Q+d_{L}\right)}$ & $\begin{array}{l}\text { time until a larva dies or is in- } \\
\text { fected (when it does not ma- } \\
\text { ture before), distributed ex- } \\
\text { ponentially }\end{array}$ \\
\hline$K_{A}$ & $d_{A} e^{-\tau d_{A}}$ & $\begin{array}{l}\text { time until an adult dies, dis- } \\
\text { tributed exponentially }\end{array}$ \\
\hline
\end{tabular}

Appendix A.1. Transition probabilities $\Pi_{1}(P)$ and $\Pi_{2}(Q)$

When the parasitoid densities are constant, the probability for a freshly laid egg to mature to a larva is

$$
\begin{aligned}
\Pi_{1}(P) & =\mathbb{P}\left[X_{E}<K_{E}\right] \\
& =\int_{0}^{\infty} \int_{\tau}^{\infty} w_{E}(\tau)\left(a_{P} P+d_{E}\right) e^{-\left(a_{P} P+d_{E}\right) \sigma} d \sigma d \tau \\
& =\int_{0}^{\infty} w_{E}(\tau) e^{-\left(a_{P} P+d_{E}\right) \tau} d \tau,
\end{aligned}
$$

where we use the independence of $X_{E}$ and $K_{E}$. Likewise the probability for a freshly hatched larva to mature to an adult is given by

$$
\Pi_{2}(Q)=\mathbb{P}\left[X_{L}<K_{L}\right]=\int_{0}^{\infty} w_{L}(\tau) e^{-\left(a_{Q} Q+d_{L}\right) \tau} d \tau .
$$

Obviously $\Pi_{1}$ and $\Pi_{2}$ decrease strictly monotonically to 0 .

\section{Appendix A.2. Expectation values for the durations of different stages}

When the parasitoid densities are constant, the expected duration of the egg stage (which is either terminated by death of the egg or maturation to a larva) is for $a_{P} P+d_{E} \neq 0$ 


$$
\begin{aligned}
\Gamma_{1}(P)= & \mathbb{E}\left[\min \left\{K_{E}, X_{E}\right\}\right] \\
= & \mathbb{E}\left[K_{E} \mid K_{E} \leq X_{E}\right] \mathbb{P}\left[K_{E} \leq X_{E}\right]+\mathbb{E}\left[X_{E} \mid X_{E}<K_{E}\right] \mathbb{P}\left[X_{E}<K_{E}\right] \\
= & \mathbb{E}\left[K_{E} \mid K_{E} \leq X_{E}\right] \mathbb{P}\left[K_{E} \leq X_{E}\right] \\
& +\left(\mathbb{E}\left[K_{E} \mid X_{E}<K_{E}\right]-\mathbb{E}\left[K_{E}-X_{E} \mid X_{E}<K_{E}\right]\right) \mathbb{P}\left[X_{E}<K_{E}\right] \\
= & \mathbb{E}\left[K_{E} \mid K_{E} \leq X_{E}\right] \mathbb{P}\left[K_{E} \leq X_{E}\right] \\
& +\left(\mathbb{E}\left[K_{E} \mid X_{E}<K_{E}\right]-\mathbb{E}\left[K_{E}\right]\right) \mathbb{P}\left[X_{E}<K_{E}\right] \\
= & \mathbb{E}\left[K_{E}\right]-\mathbb{E}\left[K_{E}\right] \mathbb{P}\left[X_{E}<K_{E}\right] \\
= & \frac{1}{a_{P} P+d_{E}}\left(1-\Pi_{1}(P)\right)
\end{aligned}
$$

where we used that $K_{E}$ is exponentially distributed.

For $a_{P} P+d_{E}=0$ obviously

$$
\Gamma_{1}(0)=\mathbb{E}\left[X_{E}\right]
$$

In the same way the expected duration of the larva stage (given that it is reached) can be calculated for constant parasitoid densities and $a_{Q} Q+d_{L} \neq 0$,

$$
\Gamma_{2}(Q)=\mathbb{E}\left[\min \left\{K_{L}, X_{L}\right\}\right]=\frac{1}{a_{Q} Q+d_{L}}\left(1-\Pi_{2}(Q)\right)
$$

and for $a_{Q} Q+d_{L}=0$

$$
\Gamma_{2}(0)=\mathbb{E}\left[X_{L}\right]
$$

Note that the expectation values of $K_{E}$ and $K_{L}$ and thus $\Gamma_{1}$ and $\Gamma_{2}$ decrease strictly monotonically with the corresponding parasitoid densities.

The expected duration of the adult stage of a freshly emerged adult is

$$
\Gamma_{3}=\mathbb{E}\left[K_{A}\right]=\frac{1}{d_{A}}
$$

\section{Appendix B. Computing $f$ for some distributions}

Elementary representations for the function $f$ from equation (21) can be found for some distribution families for the maturation delays. To facilitate the computations, we rearrange $f$ by using the formulas for $\Gamma_{1}$ and $\Gamma_{2}$ derived 
in Appendix A.2 (assuming that $a_{P} P+d_{E}$ and $a_{Q} \Pi_{2}^{-1}\left(\frac{1}{\rho \Pi_{1}(P)}\right)+d_{L}$ are non-zero),

$$
\begin{aligned}
f(P) & =\frac{\Pi_{1}(P)}{\Gamma_{1}(P)} \Gamma_{2}\left(\Pi_{2}^{-1}\left(\frac{1}{\rho \Pi_{1}(P)}\right)\right) \\
& =\frac{\Pi_{1}(P)}{\frac{1-\Pi_{1}(P)}{a_{P} P+d_{E}}} \frac{1-\Pi_{2}\left(\Pi_{2}^{-1}\left(\frac{1}{\rho \Pi_{1}(P)}\right)\right)}{a_{Q} \Pi_{2}^{-1}\left(\frac{1}{\rho \Pi_{1}(P)}\right)+d_{L}} \\
& =\left(a_{P} P+d_{E}\right) \frac{\Pi_{1}(P)}{1-\Pi_{1}(P)} \frac{1-\frac{1}{\rho \Pi_{1}(P)}}{a_{Q} \Pi_{2}^{-1}\left(\frac{1}{\rho \Pi_{1}(P)}\right)+d_{L}} .
\end{aligned}
$$

Now the following formulas for $f$ in the special cases can be easily verified.

\section{Appendix B.1. Constant durations}

The maturation from egg to larva and from larva to adult takes a constant time $T_{E}$ and $T_{L}$ respectively. For this distribution

$$
\begin{aligned}
& \Pi_{1}(P)=e^{-\left(a_{P} P+d_{E}\right) T_{E}} \\
& \Pi_{2}(Q)=e^{-\left(a_{Q} Q+d_{L}\right) T_{L}}
\end{aligned}
$$

and (for $d_{E}>0$ and $d_{L}>0$ )

$$
f(P)=\frac{T_{L}\left(a_{P} P+d_{E}\right)\left(\rho e^{-\left(a_{P} P+d_{E}\right) T_{E}}-1\right)}{\rho\left(\log (\rho)-\left(a_{P} P+d_{E}\right) T_{E}\right)\left(1-e^{-\left(a_{P} P+d_{E}\right) T_{E}}\right)} .
$$

The function $f(P)$ decreases strictly monotonically in its domain $P \in\left[0, P^{*}\right]$ with $P^{*}=\left(\log (\rho)-d_{L} T_{L}-d_{E} T_{E}\right) /\left(T_{E} a_{P}\right)$ obtained by solving (13). ${ }^{1}$ Therefore the arguments of Section 4.3 and 5 show that a coexistence equilibrium is necessarily unique and arises only when none of the parasitoids can invade an equilibrium population of the other parasitoid and the host. To prove the monotonicity of $f(P)$ we define $\gamma=\left(a_{P} P+d_{E}\right) T_{E}$ and $q=\log (\rho)$. The

\footnotetext{
${ }^{1}$ Note that for $d_{E}=0$ or $d_{L}=0$, the stated representation of $f(P)$ is undefined at the boundary of its domain but our result on monotonicity stays generally valid for the original function defined in (21). This can be verified by a simple limit argument.
} 
domain for $P$ implies that $0<\gamma<q$. Obviously $f(P)$ is decreasing if the following function $g(\gamma)$ is decreasing,

$$
g(\gamma)=e^{q} \frac{T_{E}}{T_{L}} f(P)=\frac{\gamma\left(e^{\gamma}-e^{q}\right)}{(\gamma-q)\left(e^{\gamma}-1\right)} .
$$

To prove the desired monotonicity of $g(\gamma)$, we take the derivative by $\gamma$ and show that $g_{\gamma}(\gamma)<0$ for $0<\gamma<q$. Differentiation yields

$$
g_{\gamma}(\gamma)=\frac{e^{\gamma}\left(q+q \gamma-\gamma^{2}\right)+e^{q+\gamma}\left(q-q \gamma+\gamma^{2}\right)-q e^{2 \gamma}-e^{q} q}{(\gamma-q)^{2}\left(e^{\gamma}-1\right)^{2}}
$$

and the numerator (now interpreted as a function of $q$ for any $\gamma>0$ )

$$
k(q)=e^{\gamma}\left(q+q \gamma-\gamma^{2}\right)+e^{q+\gamma}\left(q-q \gamma+\gamma^{2}\right)-q e^{2 \gamma}-e^{q} q
$$

determines the sign of $g_{\gamma}(\gamma)$. The first two derivatives of $k(q)$ by $q$ are

$$
\begin{aligned}
k_{q}(q) & =e^{q+\gamma}\left(q-q \gamma+\gamma^{2}+1-\gamma\right)-e^{q}(1+q)-e^{2 \gamma}+e^{\gamma}(\gamma+1) \\
k_{q q}(q) & =e^{q}\left(e^{\gamma}\left(q-q \gamma+\gamma^{2}-2 \gamma+2\right)-q-2\right) .
\end{aligned}
$$

It can be easily seen that the equation $k_{q q}(q)=0$ has only one solution for $q$. Therefore $k_{q}(q)=0$ has at most two solutions and $k(q)$ has at most two (local) extrema.

Moreover, we see that $k(0)=k(\gamma)=0$, that $k(q) \underset{q \rightarrow-\infty}{\longrightarrow} \infty$ (the dominant term being $q e^{\gamma}$ with coefficient $1+\gamma-e^{\gamma}$ ), and that $k(q) \underset{q \rightarrow \infty}{\longrightarrow}-\infty$ (the dominant term being $q e^{q}$ with coefficient $\left.e^{\gamma}(1-\gamma)-1\right)$. Since $k_{q}(\gamma)=0$, this implies $k(q)<0$ for $q>\gamma$ (and actually $k(q) \leq 0$ for $q \geq 0$ ). This completes the proof that $f(P)$ decreases strictly monotonically.

\section{Appendix B.2. Two-value distribution}

The maturation delay from egg to larva and from larva to adult are each distributed with two distinct values that occur with certain probabilities. The transformation from egg to larva has length $T_{E_{1}}$ with probability $r_{E}$ and length $T_{E_{2}}$ with probability $1-r_{E}$. The transformation from larva to adult has length $T_{L_{1}}$ with probability $r_{L}$ and length $T_{L_{2}}$ with probability $1-r_{L}$. For this distribution

$$
\begin{aligned}
& \Pi_{E}(P)=r_{E} e^{\left(a_{P} P+d_{E}\right) T_{E_{1}}}+\left(1-r_{E}\right) e^{\left(a_{P} P+d_{E}\right) T_{E_{2}}} \\
& \Pi_{L}(Q)=r_{L} e^{\left(a_{Q} Q+d_{L}\right) T_{L_{1}}}+\left(1-r_{L}\right) e^{\left(a_{Q} Q+d_{L}\right) T_{L_{2}}} .
\end{aligned}
$$


$\Pi_{L}^{-1}$ and therefore $f$ have no elementary representations. The numerical example presented in Fig. 1 shows however that $f$ can be non-monotonic and that therefore multiple coexistence equilibria can occur.

\section{Appendix B.3. Exponential distribution}

The maturation delays from egg to larva and from larva to adult are exponentially distributed with expectation $1 / \lambda_{E}$ and $1 / \lambda_{L}$ respectively. For this distribution

$$
\begin{aligned}
\Pi_{1}(P) & =\frac{\lambda_{E}}{a_{P} P+d_{E}+\lambda_{E}} \\
\Pi_{2}(Q) & =\frac{\lambda_{L}}{a_{Q} Q+d_{L}+\lambda_{L}}
\end{aligned}
$$

and

$$
f(P)=\frac{a_{P} P+d_{E}+\lambda_{E}}{\rho \lambda_{L}} .
$$

Obviously $f(P)$ increases strictly monotonically in this case. Therefore the arguments of Section 4.3 and 5 state that a coexistence equilibrium is necessarily unique and arises only in the case of mutual invasibility.

\section{Appendix B.4. Shifted exponential distribution}

The maturation delay from egg to larva and from larva to adult have shifted exponential distributions. They have a minimum duration of $m_{E}$ and $m_{L}$ respectively, followed by an additional time which is distributed exponentially with expectation $1 / \lambda_{E}$ and $1 / \lambda_{L}$ respectively. For this distribution

$$
\begin{aligned}
& \Pi_{E}(P)=e^{-\left(a_{P} P+d_{E}\right) m_{E}} \frac{\lambda_{E}}{a_{P} P+d_{E}+\lambda_{E}} \\
& \Pi_{L}(Q)=e^{-\left(a_{Q} Q+d_{L}\right) m_{L}} \frac{\lambda_{l}}{a_{Q} Q+d_{L}+\lambda_{L}} .
\end{aligned}
$$

$\Pi_{L}^{-1}$ and therefore $f$ have no elementary representations. Numerical calculations show that $f$ can become non-monotonous and therefore multiple equilibria can arise. 


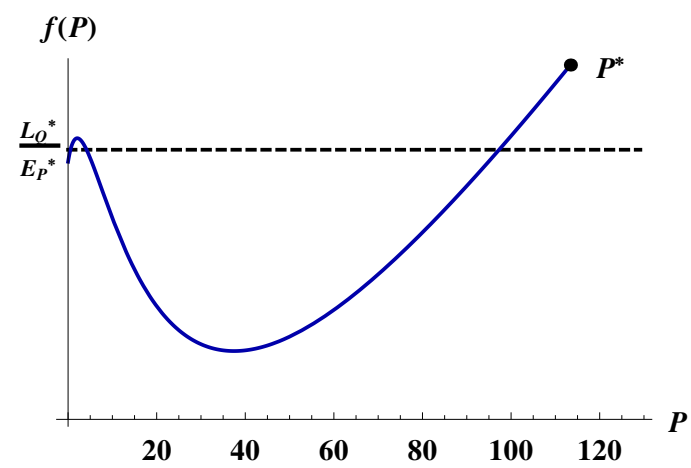

Figure B.7: The graph of the function $f$ with gamma distributed maturation delays. Parameter values are $p_{E}=2, p_{L}=5, \lambda_{E}=1, \lambda_{L}=1, a_{P}=0.198, a_{Q}=1, d_{E}=0$, $d_{L}=0, \rho=550, d_{P}=1, d_{Q}=1, c_{P}=1$ and $c_{Q}=1$

\section{Appendix B.5. Gamma distribution}

The maturation delay from egg to larva and from larva to adult have gamma distributions with shape parameter $p_{E}$ and $p_{L}$ respectively and inverse scale parameter $\lambda_{E}$ and $\lambda_{L}$ respectively. For this distribution

$$
\begin{aligned}
& \Pi_{E}(P)=\left(\frac{\lambda_{E}}{a_{P} P+d_{E}+\lambda_{E}}\right)^{p_{E}} \\
& \Pi_{L}(Q)=\left(\frac{\lambda_{L}}{a_{Q} Q+d_{L}+\lambda_{L}}\right)^{p_{L}} .
\end{aligned}
$$

$\Pi_{L}^{-1}$ and therefore $f$ have elementary representations,

$$
f(P)=\frac{\left(a_{P} P+d_{E}\right)\left(\left(a_{P} P+d_{E}+\lambda_{E}\right)^{p_{E}}-\rho \lambda_{E}^{p_{E}}\right)\left(\frac{\lambda_{E}^{-p_{E}}\left(a_{P} P+d_{E}+\lambda_{E}\right)^{p_{E}}}{\rho}\right)^{1 / p_{L}}}{\lambda_{L} \rho\left(\left(a_{P} P+d_{E}+\lambda_{E}\right)^{p_{E}}-\lambda_{E}^{p_{E}}\right)\left(\left(\frac{\lambda_{E}^{-p_{E}}\left(a_{P} P+d_{E}+\lambda_{E}\right)^{p_{E}}}{\rho}\right)^{1 / p_{L}}-1\right)} .
$$

Numerical calculations show that $f$ can become non-monotonous and therefore multiple equilibria can arise, see Fig. B.7.

\section{Appendix B.6. Shifted gamma distribution}

The maturation delay from egg to larva and from larva to adult have shifted gamma distributions. They have a minimum duration of $m_{E}$ and $m_{L}$ respectively, followed by an additional time which is gamma distributed with 
shape parameter $p_{E}$ and $p_{L}$ respectively and inverse scale parameter $\lambda_{E}$ and $\lambda_{L}$ respectively. For this distribution

$$
\begin{aligned}
& \Pi_{E}(P)=e^{-\left(a_{P} P+d_{E}\right) m_{E}}\left(\frac{\lambda_{E}}{a_{P} P+d_{E}+\lambda_{E}}\right)^{p_{E}} \\
& \Pi_{L}(Q)=e^{-\left(a_{Q} Q+d_{L}\right) m_{L}}\left(\frac{\lambda_{L}}{a_{Q} Q+d_{L}+\lambda_{L}}\right)^{p_{L}} .
\end{aligned}
$$

$\Pi_{L}^{-1}$ and therefore $f$ have no elementary representations. As with the nonshifted gamma distribution, $f$ can become non-monotonous and therefore multiple equilibria can arise.

\section{Appendix C. Characteristic equation}

Here we derive a characteristic equation by considering a small perturbation from an equilibrium $(\bar{E}, \bar{L}, \bar{A}, \bar{P}, \bar{Q})$,

$$
\begin{aligned}
& E(t)=\bar{E}+e(t), \quad L(t)=\bar{L}+l(t), A(t)=\bar{A}+a(t) \\
& P(t)=\bar{P}+p(t), \quad Q(t)=\bar{Q}+q(t)
\end{aligned}
$$

and assume that

$$
\begin{aligned}
& e(t)=h_{E} e^{\lambda t}, l(t)=h_{L} e^{\lambda t}, a(t)=h_{A} e^{\lambda t} \\
& p(t)=h_{P} e^{\lambda t}, q(t)=h_{Q} e^{\lambda t} .
\end{aligned}
$$

The aim of the characteristic equation is to investigate stability of an equilibrium by the complex roots for $\lambda$. An equilibrium is stable when all roots have negative real parts while it is unstable when there are roots with positive real part, see (Diekmann et al., 1995). In order to derive the characteristic equation, we define

$$
\begin{aligned}
\bar{R}_{E} & :=\rho d_{A} \bar{A} \\
\gamma_{E} & :=a_{P} \bar{P}+d_{E} \\
\gamma_{L} & :=a_{Q} \bar{Q}+d_{L} \\
\bar{M}_{E} & :=\int_{0}^{\infty} \bar{R}_{E} e^{-x_{E} \gamma_{E}} w_{E}\left(x_{E}\right) d x_{E} \\
\bar{M}_{L} & :=\int_{0}^{\infty} \bar{M}_{E} e^{-x_{L} \gamma_{L}} w_{L}\left(x_{L}\right) d x_{L}
\end{aligned}
$$




$$
\begin{aligned}
r_{E}(t): & =R_{E}(t)-\bar{R}_{E} \\
& =\rho d_{A} A(t)-\bar{R}_{E} \\
& =\rho d_{A}(\bar{A}+a(t))-\bar{R}_{E} \\
& =\rho d_{A} a(t)
\end{aligned}
$$

515

and

$$
\begin{aligned}
m_{E}(t):= & M_{E}(t)-\bar{M}_{E} \\
= & \int_{0}^{\infty} R_{E}\left(t-x_{E}\right) S_{E}\left(x_{E}, t\right) w_{E}\left(x_{E}\right) d x_{E}-\bar{M}_{E} \\
= & \int_{0}^{\infty}\left(\bar{R}_{E}+r_{E}\left(t-x_{E}\right)\right) e^{-x_{E} \gamma_{E}} e^{-a_{P} \int_{t-x_{E}}^{t} p(y) d y} w_{E}\left(x_{E}\right) d x_{E}-\bar{M}_{E} \\
= & \int_{0}^{\infty}\left(\bar{R}_{E}+r_{E}\left(t-x_{E}\right)\right) e^{-x_{E} \gamma_{E}}\left(1-a_{P} \int_{t-x_{E}}^{t} p(y) d y\right) w_{E}\left(x_{E}\right) d x_{E}-\bar{M}_{E} \\
= & \int_{0}^{\infty} r_{E}\left(t-x_{E}\right) e^{-x_{E} \gamma_{E}} w_{E}\left(x_{E}\right) d x_{E} \\
& -\int_{0}^{\infty} \bar{R}_{E} e^{-x_{E} \gamma_{E}} a_{P} \int_{t-x_{E}}^{t} p(y) d y w_{E}\left(x_{E}\right) d x_{E}
\end{aligned}
$$

516 where we use that $e^{x} \approx 1+x$ for small $x$ and that $r_{E}\left(t-x_{E}\right) p(y) \approx 0$. In 517 the same way

$$
\begin{aligned}
m_{L}(t):= & M_{L}(t)-\bar{M}_{L} \\
= & \int_{0}^{\infty} m_{E}\left(t-x_{L}\right) e^{-x_{L} \gamma_{L}} w_{L}\left(x_{L}\right) d x_{L} \\
& -\int_{0}^{\infty} \bar{M}_{E} e^{-x_{L} \gamma_{L}} a_{Q} \int_{t-x_{L}}^{t} q(y) d y w_{L}\left(x_{L}\right) d x_{L} \\
= & \int_{0}^{\infty}\left(\int_{0}^{\infty} r_{E}\left(t-x_{E}-x_{L}\right) e^{-x_{E} \gamma_{E}} w_{E}\left(x_{E}\right) d x_{E}\right. \\
& \left.-\int_{0}^{\infty} \bar{R}_{E} e^{-x_{E} \gamma_{E}} a_{P} \int_{t-x_{E}-x_{L}}^{t-x_{L}} p(y) d y w_{E}\left(x_{E}\right) d x_{E}\right) \cdot e^{-x_{L} \gamma_{L}} w_{L}\left(x_{L}\right) d x_{L} \\
& -\int_{0}^{\infty} \bar{M}_{E} e^{-x_{L} \gamma_{L}} a_{Q} \int_{t-x_{L}}^{t} q(y) d y w_{L}\left(x_{L}\right) d x_{L} .
\end{aligned}
$$




$$
\begin{aligned}
\dot{e}(t) & =\dot{E}(t)=R_{E}(t)-M_{E}(t)-a_{P} E(t) P(t)-d_{E} E(t) \\
& =\bar{R}_{E}+r_{E}(t)-\left(\bar{M}_{E}+m_{E}(t)\right)-a_{P}(\bar{E}+e(t))(\bar{P}+p(t))-d_{E}(\bar{E}+e(t)) \\
& =r_{E}(t)-m_{E}(t)-a_{P}(\bar{E} p(t)+e(t) \bar{P})-d_{E} e(t)
\end{aligned}
$$

$$
\begin{aligned}
\dot{l}(t) & =m_{E}(t)-m_{L}(t)-a_{Q}(\bar{L} q(t)+l(t) \bar{Q})-d_{L} l(t) \\
\dot{a}(t) & =m_{L}(t)-d_{A} a(t) \\
\dot{p}(t) & =c_{P} a_{P}\left(\bar{E} p\left(t-T_{J P}\right)+e\left(t-T_{J P}\right) \bar{P}\right)-d_{P} p(t) \\
\dot{q}(t) & =c_{Q} a_{Q}\left(\bar{L} q\left(t-T_{J Q}\right)+l\left(t-T_{J Q}\right) \bar{Q}\right)-d_{Q} q(t) .
\end{aligned}
$$

We introduce the notation

$$
\begin{aligned}
\bar{\Pi}_{1} & :=\Pi_{1}(\bar{P})=\int_{0}^{\infty} e^{-x_{E} \gamma_{E}} w_{E}\left(x_{E}\right) d x_{E} \\
\bar{\Pi}_{2} & :=\Pi_{2}(\bar{Q})=\int_{0}^{\infty} e^{-x_{L} \gamma_{L}} w_{L}\left(x_{L}\right) d x_{L} \\
\bar{\Pi}_{1}(\lambda) & :=\Pi_{1}\left(\bar{P}+\frac{\lambda}{a_{P}}\right)=\int_{0}^{\infty} e^{-x_{E}\left(\gamma_{E}+\lambda\right)} w_{E}\left(x_{E}\right) d x_{E} \\
\bar{\Pi}_{2}(\lambda) & :=\Pi_{2}\left(\bar{Q}+\frac{\lambda}{a_{Q}}\right)=\int_{0}^{\infty} e^{-x_{L}\left(\gamma_{L}+\lambda\right)} w_{L}\left(x_{L}\right) d x_{L}
\end{aligned}
$$

and obtain the following by plugging (C.2) into (C.7) and (C.8)

$$
\begin{aligned}
& \lambda h_{E}=\rho d_{A}\left(h_{A}-h_{A} \bar{\Pi}_{1}(\lambda)+\bar{A} a_{P} h_{P} \frac{\bar{\Pi}_{1}-\bar{\Pi}_{1}(\lambda)}{\lambda}\right)-a_{P}\left(\bar{E} h_{P}+h_{E} \bar{P}\right)-d_{E} h_{E} \\
& \lambda h_{L}=\rho d_{A}\left(h_{A} \bar{\Pi}_{1}(\lambda)-\bar{A} a_{P} h_{P} \frac{\bar{\Pi}_{1}-\bar{\Pi}_{1}(\lambda)}{\lambda}-h_{A} \bar{\Pi}_{1}(\lambda) \bar{\Pi}_{2}(\lambda)+\bar{A} a_{P} h_{P} \frac{\bar{\Pi}_{1}-\bar{\Pi}_{1}(\lambda)}{\lambda} \bar{\Pi}_{2}(\lambda)\right. \\
& \left.+\bar{A} a_{Q} h_{Q} \bar{\Pi}_{1} \frac{\bar{\Pi}_{2}-\bar{\Pi}_{2}(\lambda)}{\lambda}\right)-a_{Q}\left(\bar{L} h_{Q}+h_{L} \bar{Q}\right)-d_{L} h_{L} \\
& \lambda h_{A}=\rho d_{A}\left(h_{A} \bar{\Pi}_{1}(\lambda) \bar{\Pi}_{2}(\lambda)-\bar{A} a_{P} h_{P} \frac{\bar{\Pi}_{1}-\bar{\Pi}_{1}(\lambda)}{\lambda} \bar{\Pi}_{2}(\lambda)-\bar{A} a_{Q} h_{Q} \bar{\Pi}_{1} \frac{\bar{\Pi}_{2}-\bar{\Pi}_{2}(\lambda)}{\lambda}\right)-d_{A} h_{A} \\
& \lambda h_{P}=a_{P} c_{P} e^{-\lambda T_{J P}}\left(\bar{E} h_{P}+h_{E} \bar{P}\right)-d_{P} h_{P} \\
& \lambda h_{Q}=a_{Q} c_{Q} e^{-\lambda T_{J Q}}\left(\bar{L} h_{Q}+h_{L} \bar{Q}\right)-d_{Q} h_{Q}
\end{aligned}
$$


where we divide on both sides by $e^{\lambda t}$ and use that $\bar{M}_{E}=\rho d_{A} \overline{A \Pi}_{1}$. From the last two equations of (C.10) we can express $h_{p}$ and $h_{q}$ explicitly in terms of $h_{e}$ and $h_{l}$ as

$$
\begin{aligned}
h_{P} & =h_{E} \Phi_{P}(\lambda) \quad \text { where } & \Phi_{P}(\lambda) & =\frac{\bar{P} a_{P} c_{P} e^{-\lambda T_{J P}}}{\lambda+d_{P}-a_{P} c_{P} \bar{E} e^{-\lambda T_{J P}}} \\
h_{Q} & =h_{L} \Phi_{Q}(\lambda) \quad \text { where } & \Phi_{Q}(\lambda) & =\frac{\bar{Q} a_{Q} c_{Q} e^{-\lambda T_{J Q}}}{\lambda+d_{Q}-a_{Q} c_{Q} \bar{L} e^{-\lambda T_{J Q}}} .
\end{aligned}
$$

Using the solutions from (C.11) and the first two equations in (C.10) we can express $h_{E}$ and $h_{L}$ in the following form,

$h_{E}=h_{A} \Phi_{E}(\lambda)$

where

$$
\Phi_{E}(\lambda)=\frac{\rho d_{A}\left(1-\bar{\Pi}_{1}(\lambda)\right)}{\lambda+d_{E}+a_{P} \bar{P}+\Phi_{P}(\lambda)\left(a_{P} \bar{E}-\rho d_{A} \bar{A} a_{P} \frac{\bar{\Pi}_{1}-\bar{\Pi}_{1}(\lambda)}{\lambda}\right)}
$$

$h_{L}=h_{A} \Phi_{L}(\lambda)$

$$
\text { where } \quad \Phi_{L}(\lambda)=\frac{\rho d_{A}\left(\bar{\Pi}_{1}(\lambda)\left(1-\bar{\Pi}_{2}(\lambda)\right)-\Phi_{E}(\lambda) \Phi_{P}(\lambda) \bar{A} a_{P}\left(1-\bar{\Pi}_{2}(\lambda) \frac{\bar{\Pi}_{1}-\bar{\Pi}_{1}(\lambda)}{\lambda}\right)\right.}{\lambda+d_{L}+a_{Q} \bar{Q}+\Phi_{Q}(\lambda)\left(a_{Q} \bar{L}-\rho d_{A} \bar{A} a_{Q} \bar{\Pi}_{1} \frac{\bar{\Pi}_{2}-\bar{\Pi}_{2}(\lambda)}{\lambda}\right)} .
$$

Plugging $h_{P}, h_{Q}, h_{E}$ and $h_{L}$ in the third equation of (C.10) we have the characteristic equation in the form $G(\lambda)=1$,

$$
\begin{aligned}
& G(\lambda)= \\
& \frac{\rho d_{A}}{\lambda+d_{A}}\left(\bar{\Pi}_{1}(\lambda) \bar{\Pi}_{2}(\lambda)-\bar{A} a_{P} \bar{\Pi}_{2}(\lambda) \Phi_{P}(\lambda) \Phi_{E}(\lambda) \frac{\bar{\Pi}_{1}-\bar{\Pi}_{1}(\lambda)}{\lambda}-\bar{A} a_{Q} \bar{\Pi}_{1} \Phi_{Q}(\lambda) \Phi_{L}(\lambda) \frac{\bar{\Pi}_{2}-\bar{\Pi}_{2}(\lambda)}{\lambda}\right) .
\end{aligned}
$$

\section{Appendix C.1. A sufficient condition for instability}

The following observation can be helpful for proving instability of an equilibrium. It is easily verified that $G(\lambda) \underset{\lambda \rightarrow \infty}{\longrightarrow} 0$. Hence if $G(0)>1$ then there is a positive real root for the characteristic equation and the coexistence equilibrium is unstable. Therefore we investigate the structure of $G(0)$. First we see that

$$
\begin{aligned}
& \lim _{\lambda \rightarrow 0} \frac{\bar{\Pi}_{1}-\bar{\Pi}_{1}(\lambda)}{\lambda}=-\frac{d \bar{\Pi}_{1} / d \bar{P}}{a_{P}} \\
& \lim _{\lambda \rightarrow 0} \frac{\bar{\Pi}_{2}-\bar{\Pi}_{2}(\lambda)}{\lambda}=-\frac{d \bar{\Pi}_{2} / d \bar{Q}}{a_{Q}} .
\end{aligned}
$$


536

537

$$
\begin{aligned}
\Phi_{Q}(\lambda) \Phi_{L}(\lambda) & =\Phi_{Q}(\lambda) \frac{\rho d_{A}\left(\bar{\Pi}_{1}(\lambda)\left(1-\bar{\Pi}_{2}(\lambda)\right)-\Phi_{E}(\lambda) \Phi_{P}(\lambda) \bar{A} a_{P}\left(1-\bar{\Pi}_{2}(\lambda)\right) \frac{\bar{\Pi}_{1}-\bar{\Pi}_{1}(\lambda)}{\lambda}\right)}{\lambda+d_{L}+a_{Q} \bar{Q}+\Phi_{Q}(\lambda)\left(a_{Q} \bar{L}-\rho d_{A} \bar{A} a_{Q} \bar{\Pi}_{1} \frac{\bar{\Pi}_{2}-\bar{\Pi}_{2}(\lambda)}{\lambda}\right)} \\
& =\frac{\rho d_{A}\left(\bar{\Pi}_{1}(\lambda)\left(1-\bar{\Pi}_{2}(\lambda)\right)-\Phi_{E}(\lambda) \Phi_{P}(\lambda) \bar{A} a_{P}\left(1-\bar{\Pi}_{2}(\lambda)\right) \frac{\bar{\Pi}_{1}-\bar{\Pi}_{1}(\lambda)}{\lambda}\right)}{\frac{\lambda+d_{L}+a_{Q} \bar{Q}}{\Phi_{Q}(\lambda)}+\left(a_{Q} \bar{L}-\rho d_{A} \bar{A} a_{Q} \bar{\Pi}_{1} \frac{\bar{\Pi}_{2}-\bar{\Pi}_{2}(\lambda)}{\lambda}\right)} .
\end{aligned}
$$

539

Since $1 / \Phi_{Q}(\lambda) \underset{\lambda \rightarrow 0}{\longrightarrow} 0$,

$$
\lim _{\lambda \rightarrow 0} \Phi_{Q}(\lambda) \Phi_{L}(\lambda)=\frac{\rho d_{A}\left(1-\bar{\Pi}_{2}\right)\left(a_{P} \overline{E \Pi}_{1}+\rho d_{A} \overline{A \Pi}_{1}^{\prime}\right)}{\left(a_{Q} \bar{L}+\rho d_{A} \overline{A \Pi}_{1} \bar{\Pi}_{2}^{\prime}\right)\left(a_{P} \bar{E}+\rho d_{A} \overline{A \Pi}_{1}^{\prime}\right)} .
$$

540 Now $G(0)$ can be simplified,

$G(0)$

$$
\begin{aligned}
& =\rho\left(\bar{\Pi}_{1} \bar{\Pi}_{2}+\overline{A \Pi}_{2} \bar{\Pi}_{1}^{\prime} \frac{\rho d_{A}\left(1-\bar{\Pi}_{1}\right)}{a_{P} \bar{E}+\rho d_{A} \overline{A \Pi}_{1}^{\prime}}+\overline{A \Pi}_{1} \bar{\Pi}_{2}^{\prime} \frac{\rho d_{A}\left(1-\bar{\Pi}_{2}\right)\left(a_{P} \overline{E \Pi}_{1}+\rho d_{A} \overline{A \Pi}_{1}^{\prime}\right)}{\left(a_{Q} \bar{L}+\rho d_{A} \overline{A \Pi}_{1} \bar{\Pi}_{2}^{\prime}\right)\left(a_{P} \bar{E}+\rho d_{A} \overline{A \Pi}_{1}^{\prime}\right)}\right) \\
& =\rho\left(\frac{\bar{\Pi}_{2}\left(a_{P} \overline{E \Pi}_{1}+\rho d_{A} \overline{A \Pi}_{1}^{\prime}\right)}{a_{P} \bar{E}+\rho d_{A} \overline{A \Pi}_{1}^{\prime}}+\overline{A \Pi}_{1} \bar{\Pi}_{2}^{\prime} \frac{\rho d_{A}\left(1-\bar{\Pi}_{2}\right)\left(a_{P} \overline{E \Pi}_{1}+\rho d_{A} \overline{A \Pi}_{1}^{\prime}\right)}{\left(a_{Q} \bar{L}+\rho d_{A} \overline{A \Pi}_{1} \bar{\Pi}_{2}^{\prime}\right)\left(a_{P} \bar{E}+\rho d_{A} \overline{A \Pi}_{1}^{\prime}\right)}\right) \\
& =\rho \frac{\left(a_{P} \overline{E \Pi}_{1}+\rho d_{A} \overline{A \Pi}_{1}^{\prime}\right)\left(a_{Q} \overline{L \Pi}_{2}+\rho d_{A} \overline{A \Pi}_{1} \bar{\Pi}_{2}^{\prime}\right)}{\left(a_{P} \bar{E}+\rho d_{A} \overline{A \Pi}_{1}^{\prime}\right)\left(a_{Q} \bar{L}+\rho d_{A} \overline{A \Pi}_{1} \bar{\Pi}_{2}^{\prime}\right)} .
\end{aligned}
$$



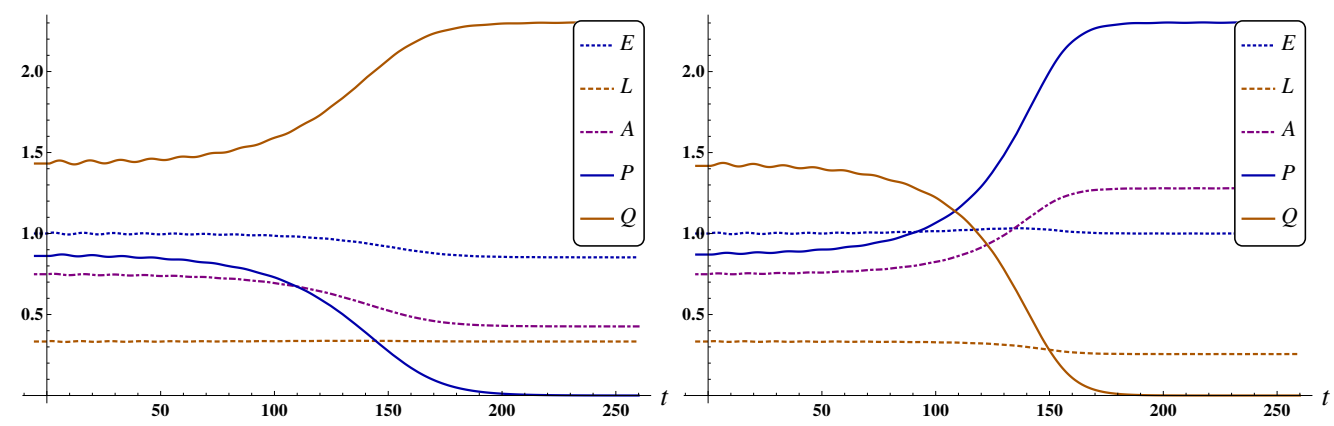

Figure C.8: Time plots of population dynamics after small perturbations from equilibrium densities. Both maturation delays, from egg to larva and from larva to adult, have constant lengths $T_{E}$ and $T_{L}$ respectively. The initial densities for $t \leq 0$ are constant and correspond to perturbations from the unique set of coexistence equilibrium densities. In the left panel, the egg parasitoid density $P$ is decreased by $1 \%$ and the larva parasitoid wins the competition. In the right panel, the larva parasitoid density $Q$ is decreased by $1 \%$ and the egg parasitoid wins the competition. Parameter values are $T_{E}=1, T_{L}=1, a_{P}=1$, $a_{Q}=1, d_{E}=0, d_{L}=0, d_{A}=0.2, \rho=10, d_{P}=1, d_{Q}=1, c_{P}=1, c_{Q}=3, T_{J P}=1$ and $T_{J Q}=1$

\section{Appendix C.2. Instability of the coexistence equilibrium when maturation de-} lays are constant

We have seen in Appendix B.1, that with constant maturation delays at most one coexistence equilibrium exists, and that if it exists, none of the parasitoids can invade an equilibrium population of the other parasitoid and the host. This observation and the simulations shown in Fig. C.8 suggest that the coexistence equilibrium is unstable. We will now prove this conjecture by using the criteria from Appendix C.1, which states that an equilibrium is unstable when the corresponding $G(0)>1$. Using the formulations of Appendix B.1 and Appendix C.1, it is easily verified that with constant maturation delays $\bar{\Pi}_{1}^{\prime}=-a_{P} T_{E} \bar{\Pi}_{1}$ and $\bar{\Pi}_{2}^{\prime}=-a_{Q} T_{L} \bar{\Pi}_{2}$. Plugging into (C.19) yields with the notation $\Gamma_{1}(\bar{P})=\bar{\Gamma}_{1}$ and $\Gamma_{2}(\bar{Q})=\bar{\Gamma}_{2}$,

$$
\begin{aligned}
G(0) & =\rho \frac{\left(a_{P} \overline{E \Pi}_{1}-a_{P} T_{E} \rho d_{A} \overline{A \Pi}_{1}\right)\left(a_{Q} \overline{L \Pi}_{2}-a_{Q} T_{L} \rho d_{A} \overline{A \Pi}_{1} \bar{\Pi}_{2}\right)}{\left(a_{P} \bar{E}-a_{P} T_{E} \rho d_{A} \overline{A \Pi}_{1}\right)\left(a_{Q} \bar{L}-a_{Q} T_{L} \rho d_{A} \overline{A \Pi}_{1} \bar{\Pi}_{2}\right)} \\
& =\frac{\bar{\Gamma}_{1}-T_{E}}{\bar{\Gamma}_{1}-T_{E} \bar{\Pi}_{1}}{\overline{\Gamma_{2}}-T_{L}-T_{L} \bar{\Pi}_{2}}^{\bar{\Gamma}_{2}},
\end{aligned}
$$


where we use $\bar{E}=\rho d_{A} \overline{A \Gamma}_{1}, \bar{L}=\rho d_{A} \overline{A \Pi}_{1} \bar{\Gamma}_{2}$ and $\rho \bar{\Pi}_{1} \bar{\Pi}_{2}=1$ according to equation (7), (8), (9) and (11). For both fractions in the last line of (C.20), the numerator is positive and the denominator is negative. To verify this, we deduce from equation (A.3) that

$$
\begin{aligned}
& \bar{\Gamma}_{1}=\mathbb{E}\left[\min \left\{K_{E}, T_{E}\right\}\right]<T_{E} \text { and } \\
& \bar{\Gamma}_{1}=\bar{\Pi}_{1} T_{E}+\left(1-\bar{\Pi}_{1}\right) \mathbb{E}\left[K_{E} \mid K_{E} \leq T_{E}\right]>\bar{\Pi}_{1} T_{E},
\end{aligned}
$$

where $K_{E}$ is an exponentially distributed random variable. In the same way $\bar{\Gamma}_{2}<T_{L}$ and $\bar{\Pi}_{2} T_{L}<\bar{\Gamma}_{2}$. To prove $G(0)>1$, it is therefore enough to show that $\bar{\Gamma}_{1}-T_{E} \bar{\Pi}_{1}<T_{E}-\bar{\Gamma}_{1}$ and $\bar{\Gamma}_{2}-T_{L} \bar{\Pi}_{2}<T_{L}-\bar{\Gamma}_{2}$. To verify the first - and in the same way the second- inequality, we use $\bar{\Gamma}_{1}=\left(1-\bar{\Pi}_{1}\right) /\left(a_{P} \bar{P}+d_{E}\right)$ from equation (A.3), and argue

$$
\begin{aligned}
\bar{\Gamma}_{1}-T_{E} \bar{\Pi}_{1} & <T_{E}-\bar{\Gamma}_{1} \Leftrightarrow \\
\frac{1-\bar{\Pi}_{1}}{a_{P} \bar{P}+d_{E}}-T_{E} \bar{\Pi}_{1} & <T_{E}-\frac{1-\bar{\Pi}_{1}}{a_{P} \bar{P}+d_{E}} \Leftrightarrow \\
1-\bar{\Pi}_{1}-\bar{\Pi}_{1}\left(a_{P} \bar{P}+d_{E}\right) T_{E} & <\left(a_{P} \bar{P}+d_{E}\right) T_{E}-1+\bar{\Pi}_{1} \Leftrightarrow \\
1-e^{-\gamma}-\gamma e^{-\gamma} & <\gamma-1+e^{-\gamma} \Leftrightarrow \\
\int_{0}^{\gamma}\left(x e^{-x}\right) d x & <\int_{0}^{\gamma}\left(1-e^{-x}\right) d x \Leftarrow \\
x e^{-x} & <1-e^{-x} \quad \forall x>0 \Leftrightarrow \\
1+x & <e^{x} \quad \forall x>0,
\end{aligned}
$$

where $\gamma=\left(a_{P} \bar{P}+d_{E}\right) T_{E}$. The last line of (C.22) is obviously true. This completes the proof that the coexistence equilibrium is unstable when the maturation delays are constant.

\section{Acknowledgment}

This research was funded by the Autonomous Province of Trento (Italy), Research funds for Grandi Progetti, Project LExEM (Laboratory of excellence for epidemiology and modeling, http://www.lexem.eu). We thank the editor and four anonymous reviewers for their comments which helped to improve the manuscript substantially. 


\section{References}

Abrams, P., 1984. Variability in resource consumption rates and the coexistence of competing species. Theoretical Population Biology 25 (1), 106-124.

Armstrong, R. A., McGehee, R., 1980. Competitive exclusion. American Naturalist $115(2), 151-170$.

Bacaër, N., Guernaoui, S., 2006. The epidemic threshold of vector-borne diseases with seasonality. Journal of mathematical biology 53 (3), 421-436.

Bate, A. M., Hilker, F. M., 2013. Predator-prey oscillations can shift when diseases become endemic. Journal of theoretical biology 316, 1-8.

Breda, D., Maset, S., Vermiglio, R., 2014. Stability of Linear Delay Differential Equations: A Numerical Approach with MATLAB. Springer.

Briggs, C., Nisbet, R., Murdoch, W., 1993. Coexistence of competing parasitoid species on a host with a variable life cycle. Theoretical Population Biology 44 (3), $341-373$.

Briggs, C. J., 1993. Competition among parasitoid species on a stage-structured host and its effect on host suppression. American Naturalist 141 (3), 372-397.

Briggs, C. J., Nisbet, R. M., Murdoch, W. W., 1999. Delayed feedback and multiple attractors in a host-parasitoid system. Journal of Mathematical Biology 38 (4), 317-345.

Buonomo, B., Cerasuolo, M., 2014. Stability and bifurcation in plant-pathogens interactions. Applied Mathematics and Computation 232, 858-871.

Chesson, P., 1994. Multispecies competition in variable environments. Theoretical Population Biology 45 (3), 227-276.

Chesson, P., 2000. Mechanisms of maintenance of species diversity. Annual review of Ecology and Systematics 31, 343-366.

Chesson, P. L., Case, T. J., 1986. Overview: nonequilibrium community theories: chance, variability, history. Community ecology. Edited by J. Diamond and TJ Case. Harper and Row Publishers, Inc., New York, 229-239.

Diekmann, O., Van Gils, S. A., Lunel, S. V., Walther, H.-O., 1995. Delay equations: functional-, complex-, and nonlinear analysis. Springer-Verlag. 
Ehler, L., 1990. Introduction strategies in biological control of insects. Critical issues in biological control/edited by Manfred Mackauer and Lester E. Ehler, Jens Roland.

Force, D. C., 1970. Competition among four hymenopterous parasites of an endemic insect host. Annals of the Entomological Society of America 63 (6), 16751688 .

Freeze, M., Chang, Y., Feng, W., 2014. Analysis of dynamics in a complex food chain with ratio-dependent functional response. Journal of Applied Analysis and Computation 4 (1), 69-87.

Gause, G., Witt, A., 1935. Behavior of mixed populations and the problem of natural selection. The American Naturalist 69 (725), 596-609.

Godfray, H., Hassell, M., 1989. Discrete and continuous insect populations in tropical environments. The Journal of Animal Ecology 58 (1), 153-174.

Godfray, H. C. J., 1994. Parasitoids: behavioral and evolutionary ecology. Princeton University Press.

Greenman, J. V., Norman, R. A., 2007. Environmental forcing, invasion and control of ecological and epidemiological systems. Journal of theoretical biology 247 (3), 492-506.

Hackett-Jones, E., Cobbold, C., White, A., 2009. Coexistence of multiple parasitoids on a single host due to differences in parasitoid phenology. Theoretical Ecology 2 (1), 19-31.

Harvey, J. A., Wagenaar, R., Martijn Bezemer, T., 2009. Life-history traits in closely related secondary parasitoids sharing the same primary parasitoid host: evolutionary opportunities and constraints. Entomologia experimentalis et applicata 132 (2), 155-164.

Hsu, S.-B., Hubbell, S., Waltman, P., 1977. A mathematical theory for singlenutrient competition in continuous cultures of micro-organisms. SIAM Journal on Applied Mathematics 32 (2), 366-383.

Lane, S. D., St. Mary, C. M., Getz, W. M., 2006. Coexistence of attack-limited parasitoids sequentially exploiting the same resource and its implications for biological control. Vol. 41. Finnish Zoological and Botanical Publishing Board, pp. 17-34. 
Li, L., Chesson, P., Bolnick, D. I., Kalisz, S., 2016. The effects of dynamical rates on species coexistence in a variable environment: The paradox of the plankton revisited. The American Naturalist 188 (2), E46-E58.

Metz, J. A. J., Nisbet, R. M., Geritz, S. A. H., 1992. How should we define "fitness" for general ecological scenarios? TREE 7 (5), 173-209.

Murdoch, W., Nisbet, R., Blythe, S., Gurney, W., Reeve, J., 1987. An invulnerable age class and stability in delay-differential parasitoid-host models. American Naturalist 129 (2), 263-282.

Murdoch, W., Nisbet, R., Luck, R., Godfray, H., Gurney, W., 1992. Size-selective sex-allocation and host feeding in a parasitoid-host model. Journal of Animal Ecology 61 (3), 533-541.

Murdoch, W. W., Briggs, C. J., Nisbet, R. M., 1997. Dynamical effects of host size-and parasitoid state-dependent attacks by parasitoids. Journal of Animal Ecology 66 (4), 542-556.

Nicholson, A. J., Bailey, V. A., 1935. The balance of animal populations.part i. Vol. 105. Blackwell Publishing Ltd, pp. 551-598.

Pedersen, B. S., Mills, N. J., 2004. Single vs. multiple introduction in biological control: the roles of parasitoid efficiency, antagonism and niche overlap. Journal of Applied Ecology 41 (5), 973-984.

Pimenov, A., Kelly, T. C., Korobeinikov, A., OCallaghan, M. J., Rachinskii, D., 2015. Adaptive behaviour and multiple equilibrium states in a predator-prey model. Theoretical population biology 101, 24-30.

Price, P. W., 1970. Characteristic permitting coexistence among parasitoids of a sawafly in Quebec. Ecology 51 (3), 445-454.

Sieber, M., Hilker, F. M., 2011. Prey, predators, parasites: intraguild predation or simpler community modules in disguise? Journal of Animal Ecology 80 (2), $414-421$. 